\title{
The prevalence and risk factors of potentially inappropriate medication use in older Chinese inpatients with multimorbidity and polypharmacy: a cross-sectional study
}

\author{
Fangyuan Tian ${ }^{\#}$, Shili Liao ${ }^{\#}$, Zhaoyan Chen, Ting Xu \\ Department of Pharmacy, West China Hospital, Sichuan University, Chengdu, China \\ Contributions: (I) Conception and design: F Tian; (II) Administrative support: T Xu; (III) Provision of study materials or patients: F Tian, S Liao; (IV) \\ Collection and assembly of data: S Liao, Z Chen; (V) Data analysis and interpretation: F Tian, S Liao; (VI) Manuscript writing: All authors; (VII) \\ Final approval of manuscript: All authors. \\ "These authors contributed equally to this work. \\ Correspondence to: Ting Xu; Fangyuan Tian. Department of Pharmacy, West China Hospital, Sichuan University, No. 37 Guoxue Lane, Chengdu \\ 610041, China. Email: tingx2009@163.com; tianfangyuan0608@163.com.
}

Background: Multimorbidity and polypharmacy are common problems among the older population
globally. They not only reduce the quality of life of older adults but also increase the prevalence of potentially
inappropriate medication (PIM) use. This study aimed to examine the prevalence and the predictors of PIMs
in hospitalized geriatric patients with multimorbidity and polypharmacy in Chengdu based on the 2015
American Geriatric Society Beers Criteria (2015 AGS Beers Criteria) and 2019 American Geriatric Society
Beers Criteria (2019 AGS Beers Criteria).

Methods: From 2016 to 2018, a cross-sectional study was conducted using electronic medical data from nine tertiary hospitals in Chengdu. The 2019 and 2015 AGS Beers Criteria were used to evaluate the PIM status of older inpatients (age $\geq 65$ years), and logistic regression was used to identify the risk factors for PIM use.

Results: A total of 17,352 inpatients were included in the study between 2016 and 2018. The prevalence of PIM use based on the 2019 AGS Beers Criteria (72.54\%) was slightly higher than that based on the 2015 AGS Beers Criteria (70.10\%). Further, the prevalence of PIM use based on the 2019 AGS Beers Criteria showed an increasing trend, from $71.17 \%$ in 2016 to $73.39 \%$ in 2018 . Logistic regression demonstrated that female, advanced age, and polypharmacy were positively associated with PIM use in older adults. The most frequently used PIMs in the inpatients were diuretics, benzodiazepines, non-steroidal anti-inflammatory drugs, antipsychotics, and selective serotonin reuptake inhibitors.

Conclusions: There is a high prevalence of PIM use in older inpatients with multimorbidity and polypharmacy in Chengdu. The 2019 AGS Beers Criteria is more sensitive for evaluating older adults in Chengdu than the 2015 AGS Beers Criteria. Further, based on the 2019 AGS Beers Criteria, the prevalence of PIM use is increasing year by year. Research on interventions rationing PIM use in the geriatric population in Chengdu are necessary in the future.

Keywords: Potentially inappropriate medications (PIM); older; inpatients; Beers criteria

Submitted Aug 02, 2021. Accepted for publication Sep 09, 2021.

doi: 10.21037/atm-21-4238

View this article at: https://dx.doi.org/10.21037/atm-21-4238

^ ORCID: 0000-0002-5187-0386. 


\section{Introduction}

China, like many countries around the world, has a rapidly growing older population. Older adults are more likely to suffer from multiple chronic non-communicable diseases requiring complex treatments, which undoubtedly has a major impact on health care and social policy planning (1). Polypharmacy, which is common in older adults and is not always avoidable, often includes the prescription of inappropriate medications and carries a risk of poor outcomes. Alterations in age-related pharmacokinetics and pharmacodynamics predispose older adults to drug-related problems (2), such as elderly patients may have altered drug responsiveness, due to reduced homeostatic reserve in different organ systems e.g. vasodilators that the risk of orthostatic hypotension is greater in older adults because of attenuated baroreceptor responses (3), a reduction in liver size and a decrease in hepatic blood flow results in reduced drug clearance e.g. propranolol (4), and age related reductions in renal function affect the clearance of drugs predominantly eliminated by the kidney e.g., penicillins, diuretics and digoxin (5). So those alterations may increase the adverse drug reactions (ADRs), increase the hospitalization and disability rates, and impose an economic burden on the social medical system (6-8).

Potentially inappropriate medications (PIMs) can be defined as medications that should be avoided in older adults due to the high risk of ADRs and/or for which there is insufficient evidence of the benefits when safer, equivalent, or more effective therapeutic alternatives are available (9). The first expert consensus on PIMs, named the Beers criteria (10), was published in 1991 by a team comprising geriatrics, pharmacy, nursing, and psychopharmacology professionals. These criteria provide a practical tool for drug management in older adults and have been widely used in drug application surveys in communities, clinics, and hospitals in multiple countries.

Some previous reports examined PIMs in geriatric patients based on the 2015 AGS Beers Criteria or 2019 AGS Beers Criteria. However, these studies mainly reported on the prevalence over one year or several months and there are a lack of data on PIM use in geriatric inpatients over three consecutive years. Therefore, in this work, we extracted prescriptions of inpatients treated in the geriatric centers of nine medical institutions in Chengdu, China from 2016 to 2018. PIMs were screened based on the 2015 AGS Beers Criteria and 2019 AGS Beers Criteria, and the risk factors for PIMs were explored. The study aimed to compare the prevalence of PIM use in older Chinese inpatients in three consecutive years based on the AGS Beers Criteria and research risk factors for PIMs. We present the following article in accordance with the STROBE reporting checklist (available at https://dx.doi. org/10.21037/atm-21-4238).

\section{Methods}

\section{Setting and sample}

A cross-sectional study was carried out in nine tertiary hospitals in Chengdu.

Through a hospital prescription analysis cooperation project led by the Chinese Pharmaceutical Association, the electronic medical records of older (aged 65 and above) inpatients with multimorbidity and polypharmacy were randomly selected from the geriatric departments of the nine hospitals between January 1, 2016 and December 31, 2018. Records with missing or incomplete information about diagnosis, sex, route of administration, or medication dosage were excluded from the study.

\section{Data collection}

Data on age, sex, diagnosis, and medication were extracted from the patients' electronic medical records, and data on patients who only received $0.9 \%$ sodium chloride and sterile water were excluded.

\section{Evaluation criteria}

PIM use in the geriatric inpatients was evaluated using the 2019 and 2015 AGS Beers Criteria, which include a total of $101 \mathrm{drug}$ standards across 5 categories $(11,12)$. Two researchers independently reviewed the medications of each patient and assessed PIMs. Any inconsistencies between the two researchers would be resolved with the help of a third professional.

\section{Statistical analysis}

All data were summarized, sorted, and analyzed using Microsoft Excel, and statistical analyses were conducted using SPSS version 23.0 (Armonk, NY: IBM Corp.).

The statistical significance level was set at 0.05 . Descriptive statistics were used to describe the study population. Continuous data were expressed as mean \pm 
standard deviation (SD); non-parametric variables were expressed as median \pm interquartile range (IQR); categorical data were presented as numbers and percentages. The $\chi^{2}$ test was employed for comparisons of differences in the distributions of categorical variables. The associations between PIMs and potential risk factors were examined through a multivariate logistic regression analysis to determine the influence of sex, age, polypharmacy, and chronic conditions on PIM-related admissions. The results of the regression analysis were presented with odds ratios (ORs) and $95 \%$ confidence intervals (CIs). P value $<0.05$ was considered to be statistically significant.

\section{Ethics approval}

This study was approved by the West China Hospital Research Ethics Board (2020/651). All procedures performed in this study conformed to the standards of the Helsinki Declaration (as revised in 2013) and subsequent relevant ethics. Due to the requirement for data to be anonymized, the individual patients could not be asked for consent to participate; therefore, we applied for an exemption to the requirement of informed consent.

\section{Results}

\section{Prescription characteristics}

The prescription characteristics of the patients in this study are depicted in Table 1. A total of 17,352 patients were included, more than half $(68.91 \%)$ of whom were male. The patients ranged from 65 to 106 years old and had a mean age (SD) of 85.66 years (7.02), with those over the age of 85 accounting for the majority (66.58\%) of the study population. The median number of medical diagnoses was 3 (IQR $=2-8)$, and $37.63 \%(n=6,530)$ of patients reported having 5 or more health problems. Of the patients in this study, 36.69\% had hypertension, $29.18 \%$ had coronary heart disease (CHD), $22.50 \%$ had cerebral infarction, $21.46 \%$ had chronic obstructive pulmonary disease (COPD), and $20.18 \%$ had diabetes. Regarding pharmacotherapy, the median number of medications used was 9 (IQR $=7-11)$, and $32.91 \%(n=5,711)$ of patients were prescribed more than 10 medications. The use of at least one PIM was verified in $72.54 \%$ of patients using the 2019 AGS Beers Criteria, which was slightly higher than the proportion when using the 2015 AGS Beers Criteria (70.10\%).

\section{Details of PIMs}

We investigated PIM use in older Chinese inpatients in the Chengdu area using the AGS Beers Criteria (Table 2). A total of 17,352 patients were included in this 3-year continuous study. The prevalence of PIM use according to 2019 AGS Beers Criteria slowly increased among the older adults in our study, from $71.17 \%$ in 2016 to $73.39 \%$ in 2018

Based on the 2015 AGS Beers Criteria, 12,163 of the 17,352 patients were using PIMs, and 22,986 medications were detected. According to the 2019 AGS Beers Criteria, 12,588 of the 17,352 patients were using PIMs, and 24,064 medications were detected (Table 3). Of the patients in the PIM group, more than $68 \%$ were using 1-2 PIMs, $23 \%$ were using 3-4 PIMs, and approximately $8 \%$ were using at least 5 PIMs according to the two sets of criteria.

The top 10 PIM categories recognized by the two sets of criteria are shown in Table 4. The most frequently used PIMs were diuretics (43.73\%, 41.77\%), followed by benzodiazepines $(19.48 \%, 18.61 \%)$, non-steroidal anti-inflammatory drugs (NSAIDs) (10.53\%, 12.18\%), antipsychotics $(5.90 \%, 5.63 \%)$, and selective serotonin reuptake inhibitors (SSRIs) $(4.55 \%, 4.34 \%)$.

\section{Comparison between the two sets of criteria}

As the latest version of the AGS Beers Criteria, the 2019 version excludes some uncommon medications included in the 2015 version and adds specific medications (e.g., glimepiride, rivaroxaban, and tramadol) considered inappropriate for the older adults (Tables 5 and 6 ). Specifically, 96 PIMs, mainly related to chronic seizures or epilepsy, insomnia, and vasodilators, were removed from the 2015 AGS Beers Criteria, and 1,047 PIMs, mainly related to glimepiride, tramadol, and opioids-benzodiazepines interactions, were added to the 2015 AGS Beers Criteria.

\section{Risk factors for PIM use}

As shown in Table 1, the results of univariate analysis revealed age, sex, the number of diseases, the number of medications, and chronic diseases including CHD, COPD, diabetes, heart failure, atrial fibrillation, osteoporosis, and benign prostatic hyperplasia to be related to PIM use $(\mathrm{P}<0.05)$.

In the multivariate logistic regression model, based on the 2015 and 2019 versions of the AGS Beers Criteria, PIM 
Table 1 Basic characteristics of older patients

\begin{tabular}{|c|c|c|c|c|c|c|c|}
\hline Characteristics & Total & \multicolumn{3}{|c|}{2015 AGS Beers Criteria } & \multicolumn{3}{|c|}{2019 AGS Beers Criteria } \\
\hline$N(\%)$ & 17,352 & $5,189(29.90)$ & $12,163(70.10)$ & & $4,764(27.46)$ & $12,588(72.54)$ & \\
\hline Sex, n (\%) & & & & $<0.001$ & & & $<0.001$ \\
\hline Male & $11,957(68.91)$ & $3,824(73.69)$ & $8,133(66.87)$ & & $3,529(74.08)$ & $8,428(66.95)$ & \\
\hline Age, years (mean $\pm S D), n(\%)$ & $85.66 \pm 7.02$ & & & $<0.001$ & & & $<0.001$ \\
\hline $65-74$ & $1,572(9.06)$ & $771(14.86)$ & $801(6.59)$ & & $623(13.08)$ & $949(7.54)$ & \\
\hline $75-84$ & $4,227(24.36)$ & $1,492(28.75)$ & 2,735 (22.49) & & $1,286(26.99)$ & $2,941(23.36)$ & \\
\hline$\geq 85$ & $11,553(66.58)$ & 2,926 (56.39) & $8,627(70.93)$ & & $2,855(59.93)$ & $8,698(69.10)$ & \\
\hline$\geq 5$ & $6,530(37.63)$ & $1,687(32.51)$ & $4,843(39.82)$ & & $1,596(33.50)$ & 4,934 (39.20) & \\
\hline No. of medications [IQR], n (\%) & $9[7-11]$ & & & $<0.001$ & & & $<0.001$ \\
\hline $5-7$ & $5,741(33.09)$ & $2,588(49.87)$ & $3,153(25.92)$ & & $2,390(50.17)$ & $3,351(26.62)$ & \\
\hline $8-10$ & $5,900(34.00)$ & 1,729 (33.32) & $4,171(34.29)$ & & $1,592(33.42)$ & 4,308 (34.22) & \\
\hline$>10$ & $5,711(32.91)$ & $872(16.80)$ & $4,839(39.78)$ & & $782(16.41)$ & 4,929 (39.16) & \\
\hline \multicolumn{8}{|l|}{ Type of chronic disease, n (\%) } \\
\hline Hypertension & 6,366 (36.69) & $1,801(34.71)$ & $4,565(37.53)$ & 0.648 & $1,645(34.53)$ & $4,721(37.50)$ & 0.884 \\
\hline $\mathrm{CHD}$ & $5,063(29.18)$ & $1,171(22.57)$ & $3,892(32.00)$ & $<0.001$ & $1,023(21.47)$ & 4,040 (32.09) & $<0.001$ \\
\hline Osteoporosis & $1,762(10.15)$ & $490(9.44)$ & $1,272(1046)$ & 0.007 & $481(10.10)$ & $1,281(10.18)$ & $<0.001$ \\
\hline Benign prostatic hyperplasia & $1,420(8.18)$ & 487 (9.39) & $933(7.67)$ & $<0.001$ & $457(9.59)$ & $963(7.65)$ & $<0.001$ \\
\hline Dementia or cognitive impairment & $1,337(7.71)$ & $328(6.32)$ & $1,009(8.30)$ & 0.077 & $319(6.70)$ & $1,018(8.09)$ & 0.236 \\
\hline
\end{tabular}

AGS, American Geriatric Society; PIM, potentially inappropriate medication; SD, standard deviation; IQR, interquartile range; CHD, coronary heart disease; COPD, chronic obstructive pulmonary disease.

use was associated with age, female sex, and the number of prescribed medications, and PIM use was also associated with CHD, heart failure, and atrial fibrillation (Table 7).

\section{Discussion}

Our study reported and compared the prevalence of PIM use in older Chinese inpatients assessed using the 2015 AGS Beers Criteria and 2019 AGS Beers Criteria, and further identified the factors associated with PIM use. The major findings were as follows: Firstly, high prevalence rates of PIMs were detected $(70.10 \%$ and $72.54 \%$ using the 2015 and 2019 AGS Beers Criteria, respectively). Secondly, the prevalence of PIM use in the older Chinese inpatients 
Table 2 The prevalence of PIM use in older Chinese inpatients over a 3-year period

\begin{tabular}{lccccc}
\hline \multirow{2}{*}{ Year } & \multicolumn{2}{c}{2015 AGS Beers Criteria } & & \multicolumn{2}{c}{ 2019 AGS Beers Criteria } \\
\cline { 2 - 3 } \cline { 5 - 6 } & Non-PIM group, $n(\%)$ & PIM group, $n(\%)$ & & Non-PIM group, $n(\%)$ & PIM group, $n(\%)$ \\
\hline $2016(\mathrm{~N}=6,330)$ & $1,982(31.31)$ & $4,348(68.69)$ & & $1,825(28.83)$ & $4,505(71.17)$ \\
$2017(\mathrm{~N}=4,904)$ & $1,418(28.92)$ & $3,486(71.08)$ & & $1,311(26.73)$ & $3,593(73.27)$ \\
$2018(\mathrm{~N}=6,118)$ & $1,789(29.24)$ & $4,329(70.76)$ & & $1,628(26.61)$ & $4,490(73.39)$ \\
\hline
\end{tabular}

PIM, potentially inappropriate medication; AGS, American Geriatric Society.

Table 3 The number of PIMs used by older Chinese inpatients in the PIM group

\begin{tabular}{lcc}
\hline Characteristics & 2015 AGS Beers Criteria $(\mathrm{N}=12,163)$ & 2019 AGS Beers Criteria (N=12,588) \\
\hline PIMs, $n(\%)$ & 22,986 & 24,064 \\
1-2 PIMs & $8,343(68.59)$ & $8,585(68.20)$ \\
3-4 PIMs & $2,853(23.46)$ & $2,865(22.76)$ \\
$\geq 5$ PIMs & $967(7.95)$ & $1,138(9.04)$ \\
\hline
\end{tabular}

PIM, potentially inappropriate medication.

Table 4 Top 10 PIMs used by the older Chinese inpatients in this study

\begin{tabular}{|c|c|c|c|c|}
\hline Number & \multicolumn{2}{|c|}{2015 AGS Beers Criteria (N=22,986) } & \multicolumn{2}{|c|}{2019 AGS Beers Criteria $(\mathrm{N}=24,064)$} \\
\hline 1 & Diuretics & $10,052(43.73)$ & Diuretics & $10,052(41.77)$ \\
\hline 2 & Benzodiazepines & $4,478(19.48)$ & Benzodiazepines & $4,478(18.61)$ \\
\hline 3 & NSAIDs & $2,420(10.53)$ & NSAIDs & $2,930(12.18)$ \\
\hline 5 & SSRls & $1,045(4.55)$ & SSRIs & $1,045(4.34)$ \\
\hline 6 & Megestrol & $684(2.98)$ & Megestrol & $684(2.84)$ \\
\hline 7 & Nonbenzodiazepine & $438(1.91)$ & Opioids & $599(2.49)$ \\
\hline 8 & Insulin & $348(1.51)$ & Nonbenzodiazepine & $438(1.82)$ \\
\hline
\end{tabular}

PIM, potentially inappropriate medication; NSAID, non-steroidal anti-inflammatory drug; SSRI, selective serotonin reuptake inhibitor.

gradually increased over three consecutive years according to 2019 AGS Beers Criteria. Thirdly, with both sets of criteria, diuretics, benzodiazepines, NSAIDs, antipsychotics, and SSRIs were the most frequently used PIMs. Finally, we also found that female sex, old age, and polypharmacy were common risk factors for PIM use in the older inpatients.

With the advent of the aging population in China, the government is increasingly paying attention to rational medication use in older patients. Based on the 2015 and 2019 AGS Beers Criteria, this study found that the prevalence of PIM use among older inpatients in Chengdu was $70.10 \%$ and $72.54 \%$, respectively, which was similar to the prevalence of $71.9 \%$ reported by a study on Japanese inpatients according to the 2015 AGS Beers Criteria (13). A slightly lower prevalence of PIM use was observed among Indian inpatients using the 2015 and 2019 AGS Beers 
Table 5 The medications/criteria included in the 2015 AGS Beers Criteria but not in the 2019 AGS Beers Criteria

\begin{tabular}{lcc}
\hline Item & Medications & Total \\
\hline $\begin{array}{l}\text { Drug-disease or drug-syndrome } \\
\text { interactions }\end{array}$ & & \\
Chronic seizures or epilepsy & Olanzapine & 31 \\
& Tramadol & 2 \\
Dementia or cognitive impairment & Famotidine & 8 \\
Insomnia & Theophylline & 17 \\
Drugs that should be used with & & \\
caution & & 1 \\
& Cyclophosphamide & 17 \\
Total & Vasodilators & 37 \\
\hline
\end{tabular}

AGS, American Geriatric Society.

Table 6 The medications/criteria included in the 2019 AGS Beers Criteria but not in the 2015 AGS Beers Criteria

\begin{tabular}{|c|c|c|}
\hline Item & Medications & Total \\
\hline \multicolumn{3}{|c|}{ Medications that should be avoided } \\
\hline Sulfonylureas & Glimepiride & 332 \\
\hline \multicolumn{3}{|c|}{$\begin{array}{l}\text { Medications that should be used with } \\
\text { caution }\end{array}$} \\
\hline & Rivaroxaban & 41 \\
\hline & Tramadol & 424 \\
\hline \multicolumn{3}{|c|}{ Drug-drug interactions } \\
\hline \multirow[t]{2}{*}{ Opioids } & Benzodiazepines & 249 \\
\hline & Gabapentin & 1 \\
\hline Total & & 1047 \\
\hline
\end{tabular}

AGS, American Geriatric Society.

Criteria (60.1\% and 61.9\%, respectively) (14), while studies in Nigeria, South Africa, and Pakistan reported a prevalence of $32.1 \%, 30.1 \%$, and $36.4 \%$, respectively, based on the 2015 AGS Beers Criteria $(15,16)$. Compared with other studies in China, our result was similar to that of a study at West China Hospital in Chengdu (72.43\%) (17), but higher than those reported by studies at Beijing Chaoyang Hospital and Nanjing Drum Tower Hospital (53.5\% and 64.3\%, respectively) $(18,19)$. The universality of PIM use depends on the characteristics of patient and local prescription.
In the study the elderly inpatients were usually in serious condition with multiple diseases, and the serious condition requires to use multiple drugs. Another potential reason for the high prevalence of PIM use is that the number of prescribed medications was highly associated with PIM, and the willingness of inpatients to take drugs were relatively stronger, so they were willing to be prescribed a variety of drugs. A close association between multimorbidity or polypharmacy and PIMs has been reported in multiple studies (20-22).

Further, the prevalence of PIM use among inpatients is higher than that reported for outpatients $(30.98 \%$ and $34.39 \%$ ) in another study by our research group (23). In our research, the most common chronic conditions in older hospitalized patients were hypertension, CHD, cerebral infarction, COPD, and diabetes, however, there were slight differently in outpatients, the hypertension, CHD, diabetes, sleep disorder and hyperlipidemia were the most common chronic conditions in older outpatients. Furthermore, there were slight differently in PIM use, the most frequent PIMs in older hospitalized patients were diuretics, benzodiazepines, NSAIDs, and the benzodiazepines, diuretics, SSRIs in outpatients (23).

Our results revealed the most frequently used PIMs to be diuretics, which belong to the third part of the AGS Beers Criteria. In older patients, diuretics are recommended to be cautiously used according to need and maintained at as low a dose as possible due to their tendency to exacerbate or induce syndrome of inappropriate antidiuretic hormone secretion (SIADH) or hyponatremia $(24,25)$. Additionally, diuretics can contribute to several complications, including fractures, falls, and hyperkalemia $(26,27)$. When starting or changing diuretics doses in an older adult, the patient's electrolyte levels should be monitored closely.

The second most common class of PIMs was benzodiazepines. With age, older adults may develop sleep disorders such as difficulty falling asleep, waking up early, waking up frequently at night, or sleeping upside down. Consequently, benzodiazepines are frequently used by older patients. However, they are also associated with increased risks of cognitive impairment, delirium, falls, fractures, and motor vehicle accidents among older adults (28-31). Machado-Duque et al.'s study found that benzodiazepine use in the previous month was significantly associated with a greater probability of suffering a fall with hip fracture (32). Therefore, clinicians should minimize the use of benzodiazepines.

NSAIDs are commonly used to treat fever, headache, 
Table 7 Multivariate logistic regression analysis of factors associated with PIM use

\begin{tabular}{|c|c|c|c|c|c|c|}
\hline Characteristics & \multicolumn{3}{|c|}{2015 AGS Beers Criteria } & \multicolumn{3}{|c|}{2019 AGS Beers Criteria } \\
\hline \multicolumn{7}{|l|}{ Sex } \\
\hline Male & \multicolumn{2}{|c|}{ Reference } & \multicolumn{4}{|c|}{ Reference } \\
\hline Female & 1.515 & $1.397-1.643$ & $<0.001$ & 1.519 & $1.399-1.65$ & $<0.001$ \\
\hline \multicolumn{7}{|l|}{ Age } \\
\hline $65-74$ & \multicolumn{2}{|c|}{ Reference } & \multicolumn{4}{|c|}{ Reference } \\
\hline $75-84$ & 1.659 & $1.467-1.878$ & $<0.001$ & 1.403 & $1.236-1.592$ & $<0.001$ \\
\hline$\geq 85$ & 2.501 & $2.229-2.807$ & $<0.001$ & 1.738 & $1.545-1.955$ & $<0.001$ \\
\hline \multicolumn{7}{|l|}{ No. of diseases } \\
\hline \multicolumn{7}{|l|}{ No. of medications } \\
\hline $5-7$ & \multicolumn{2}{|c|}{ Reference } & \multicolumn{4}{|c|}{ Reference } \\
\hline $8-10$ & 1.948 & $1.799-2.108$ & $<0.001$ & 1.924 & $1.776-2.085$ & $<0.001$ \\
\hline$>10$ & 4.527 & $4.125-4.968$ & $<0.001$ & 4.577 & $4.159-5.038$ & $<0.001$ \\
\hline \multicolumn{7}{|l|}{ Type of chronic disease } \\
\hline $\mathrm{CHD}$ & 1.316 & $1.211-1.43$ & $<0.001$ & 1.476 & $1.354-1.608$ & $<0.001$ \\
\hline COPD & 0.906 & $0.83-0.988$ & 0.025 & 0.897 & $0.822-0.98$ & 0.016 \\
\hline Diabetes & - & - & - & 1.178 & $1.074-1.291$ & $<0.001$ \\
\hline
\end{tabular}

PIM, potentially inappropriate medication; AGS, American Geriatric Society; OR, odds ratio; CI, confidence interval; CHD, coronary heart disease; COPD, chronic obstructive pulmonary disease.

pain associated with inflammation in rheumatoid arthritis, or osteoarthritis in older adults. However, the most common ADRs associated with NSAID use are gastrointestinal complications, including ulcers and bleeding and renovascular/cardiovascular reactions with fluid retention, with the risk being higher in older adults. Furthermore, NSAIDs can potentially induce heart failure and increase the risk of acute kidney injury and further decline of renal function in patients with chronic kidney disease of stage IV or less. Polypharmacy is common in older adults which could increase the risk of drug-drug interactions, so it is necessary to pay attention to this. For instance, NSAIDs combined with SSRIs, warfarin, and a systemic glucocorticoid has been shown to significantly enhance the risk of upper gastrointestinal toxicity, so this combination should be avoided in older adults $(12,29,30)$.

In addition to polypharmacy, risk factors for PIM use in the older inpatients also include female sex. Female sex was significantly associated with PIM use, and this finding was consistent with various published studies $(33,34)$. It has been suggested that this might be due to females being at a higher risk for suffering from multiple chronic conditions compared to males and consequently being more susceptible for drug-disease and drug-drug interactions which will lead 
to more inappropriate prescribing (35-37). Therefore, old females need periodic medication review as a high priority.

The widely known explicit criteria for PIM is Beers Criteria developed in the United States, however, several other criteria have been elaborated or adapted to reflect national realities due to discrepancies found among countries in the factors influencing drug prescription. But, currently commonly used are the American Beers Criteria and the European STOPP/START criteria $(10,38)$, and the AGS Beers criteria suggests that the drugs should avoid and the drugs to avoid under certain diseases in elderly patients, and the STOPP/START criteria suggests the drugs that should be stopped and started according to the patient's condition. The common drugs in elderly patients are cardiovascular drugs and neurological drugs, which may aggravate the dementia or cognitive impairment of elderly inpatients, increase the risk of falls, and the readmission rate $(12,38)$. In this situation, our research focuses on finding ways to reduce the PIM use. The Reeve's study proposed the deprescribing definition first time in 2015, defined as 'Deprescribing is the process of withdrawal of an inappropriate medication, supervised by a health care professional with the goal of managing polypharmacy and improving outcomes' (39), and in order to ensure deprescribing is effective in clinical practice, it is necessary to involve a multidisciplinary team to identify the potential harm of the medication to the patient which the role of the pharmacists is defined and supported in reviewing, monitoring and optimizing medication therapy in collaboration with the physicians. And the computerassisted decision-support systems could be developed for electronic prescribing with alerts for PIM $(40,41)$. Furthermore, encouraging elderly patients to try nondrug methods of therapy is a good way, such as exercise, psychotherapy and diet. The other emphasis is exploring the relationship between PIM and ADRs and outcomes, although it has been researching, there is a lack of highquality evidence (42-44). In addition to paying attention to the effects of diseases and drugs on elderly patients, we should also pay attention to potential risk factors during hospitalization of elderly patients, such as fall, delirium and sleep disorder, so appropriate public facilities, professional nursing technology, and multidisciplinary teamwork can effectively ensure the safety of hospitalized elderly people and improve the quality of life (45).

Although this study's research sample was large and involved data from 9 hospitals in Chengdu, it still had some deficiencies due to the limitations of the research type and data. First, it was a retrospective, observational, singlecenter study conducted in Chengdu, China, which is likely to cause some deviations in the results. The result needs to be further confirmed by multi-center clinical trials, and it also means that we are unable to apply our results to other countries or hospitals. Second, the patients' detailed relevant medical records were limited, and personal information and out-of-hospital data, such as weight, smoking history, past medical history, current medical history, medication history, and ADR occurrence history, could not be obtained. Consequently, we failed to assess the associations of these factors with PIMs.

\section{Conclusions}

This study investigated the use of PIMs in older Chinese inpatients with multimorbidity and polypharmacy in Chengdu between 2016 and 2018 based on AGS Beers Criteria. Compared with the 2015 AGS Beers Criteria, the 2019 criteria were more sensitive to identifying PIMs. Furthermore, the results based on the 2019 AGS Beers Criteria showed that the prevalence of PIM use gradually increased from 2016 to 2018, and advanced age, female sex, and polypharmacy were high-risk factors for PIMs. The most frequently used PIMs were diuretics, benzodiazepines, NSAIDs, antipsychotics, and SSRIs. Together, our results show that effective intervention and management of PIM use should be conducted in elderly patients in the future.

\section{Acknowledgments}

We would like to thank the patients who participated in this study.

Funding: This work was supported by the National Key R\&D Program of China (2020 YFC2008302, 2018 YFC2002103) and Sichuan Science and Technology Program (Project No.: 2020 JDR0143 and 2020 JDR0144).

\section{Footnote}

Reporting Checklist: The authors have completed the STROBE reporting checklist. Available at https://dx.doi. org/10.21037/atm-21-4238

Data Sharing Statement: Available at https://dx.doi. org/10.21037/atm-21-4238

Conflicts of Interest: All authors have completed the ICMJE 
uniform disclosure form (available at https://dx.doi. org/10.21037/atm-21-4238). The authors have no conflicts of interest to declare.

Ethical Statement: The authors are accountable for all aspects of the work in ensuring that questions related to the accuracy or integrity of any part of the work are appropriately investigated and resolved. This study was approved by the West China Hospital Research Ethics Board (2020/651). All procedures performed in this study conformed to the standards of the Helsinki Declaration (as revised in 2013) and subsequent relevant ethics. Due to the requirement for data to be anonymized, the individual patients could not be asked for consent to participate; therefore, we applied for an exemption to the requirement of informed consent.

Open Access Statement: This is an Open Access article distributed in accordance with the Creative Commons Attribution-NonCommercial-NoDerivs 4.0 International License (CC BY-NC-ND 4.0), which permits the noncommercial replication and distribution of the article with the strict proviso that no changes or edits are made and the original work is properly cited (including links to both the formal publication through the relevant DOI and the license). See: https://creativecommons.org/licenses/by-nc-nd/4.0/.

\section{References}

1. Cojutti P, Arnoldo L, Cattani G, et al. Polytherapy and the risk of potentially inappropriate prescriptions (PIPs) among elderly and very elderly patients in three different settings (hospital, community, long-term care facilities) of the Friuli Venezia Giulia region, Italy: are the very elderly at higher risk of PIPs? Pharmacoepidemiol Drug Saf 2016;25:1070-8.

2. Davies EA, O'Mahony MS. Adverse drug reactions in special populations - the elderly. Br J Clin Pharmacol 2015;80:796-807.

3. Mangoni AA, Jackson SH. Age-related changes in pharmacokinetics and pharmacodynamics: basic principles and practical applications. Br J Clin Pharmacol 2004;57:6-14.

4. Schmucker DL. Liver function and phase I drug metabolism in the elderly: a paradox. Drugs Aging 2001;18:837-51.

5. Dreisbach AW, Lertora JJ. The effect of chronic renal failure on drug metabolism and transport. Expert Opin
Drug Metab Toxicol 2008;4:1065-74.

6. Oscanoa TJ, Lizaraso F, Carvajal A. Hospital admissions due to adverse drug reactions in the elderly. A metaanalysis. Eur J Clin Pharmacol 2017;73:759-70.

7. Hamilton H, Gallagher P, Ryan C, et al. Potentially inappropriate medications defined by STOPP criteria and the risk of adverse drug events in older hospitalized patients. Arch Intern Med 2011;171:1013-9.

8. Cahir C, Bennett K, Teljeur C, et al. Potentially inappropriate prescribing and adverse health outcomes in community dwelling older patients. Br J Clin Pharmacol 2014;77:201-10.

9. Fick DM, Cooper JW, Wade WE, et al. Updating the Beers criteria for potentially inappropriate medication use in older adults: results of a US consensus panel of experts. Arch Intern Med 2003;163:2716-24.

10. Beers MH, Ouslander JG, Rollingher I, et al. Explicit criteria for determining inappropriate medication use in nursing home residents. UCLA Division of Geriatric Medicine. Arch Intern Med 1991;151:1825-32.

11. By the American Geriatrics Society 2015 Beers Criteria Update Expert Panel. American Geriatrics Society 2015 Updated Beers Criteria for Potentially Inappropriate Medication Use in Older Adults. J Am Geriatr Soc 2015;63:2227-46.

12. By the 2019 American Geriatrics Society Beers Criteria ${ }^{\circledR}$ Update Expert Panel. American Geriatrics Society 2019 Updated AGS Beers Criteria ${ }^{\circledR}$ for Potentially Inappropriate Medication Use in Older Adults. J Am Geriatr Soc 2019;67:674-94.

13. Huang $\mathrm{CH}$, Umegaki $\mathrm{H}$, Watanabe $\mathrm{Y}$, et al. Potentially inappropriate medications according to STOPP-J criteria and risks of hospitalization and mortality in elderly patients receiving home-based medical services. PLoS One 2019;14:e211947.

14. Sharma R, Bansal P, Garg R, et al. Prevalence of potentially inappropriate medication and its correlates in elderly hospitalized patients: A cross-sectional study based on Beers criteria. J Family Community Med 2020;27:200-7.

15. Saka SA, Nlooto M, Oosthuizen F. American Geriatrics Society-Beers Criteria and adverse drug reactions: a comparative cross-sectional study of Nigerian and South African older inpatients. Clin Interv Aging 2018;13:2375-87.

16. Sarwar MR, Dar AR, Mahar SY, et al. Assessment of prescribing potentially inappropriate medications listed in Beers criteria and its association with the unplanned 
hospitalization: a cross-sectional study in Lahore, Pakistan. Clin Interv Aging 2018;13:1485-95.

17. Li H, Pu S, Liu Q, et al. Potentially inappropriate medications in Chinese older adults: The beers criteria compared with the screening tool of older persons' prescriptions criteria. Geriatr Gerontol Int 2017;17:1951-8.

18. Zhang X, Zhou S, Pan K, et al. Potentially inappropriate medications in hospitalized older patients: a cross-sectional study using the Beers 2015 criteria versus the 2012 criteria. Clin Interv Aging 2017;12:1697-703.

19. He D, Zhu H, Zhou H, et al. Potentially inappropriate medications in Chinese older adults: a comparison of two updated Beers criteria. Int J Clin Pharm 2021;43:229-35.

20. Chen LL, Tangiisuran B, Shafie AA, et al. Evaluation of potentially inappropriate medications among older residents of Malaysian nursing homes. Int J Clin Pharm 2012;34:596-603.

21. Hasan SS, Kow CS, Verma RK, et al. An evaluation of medication appropriateness and frailty among residents of aged care homes in Malaysia: A cross-sectional study. Medicine (Baltimore) 2017;96:e7929.

22. Morin L, Laroche ML, Texier G, et al. Prevalence of Potentially Inappropriate Medication Use in Older Adults Living in Nursing Homes: A Systematic Review. J Am Med Dir Assoc 2016;17:862.e1-9.

23. Tian F, Li H, Chen Z, et al. Potentially inappropriate medications in Chinese older outpatients in tertiary hospitals according to Beers criteria: A cross-sectional study. Int J Clin Pract 2021;75:e14348.

24. Kojima T, Mizukami K, Tomita N, et al. Screening Tool for Older Persons' Appropriate Prescriptions for Japanese: Report of the Japan Geriatrics Society Working Group on "Guidelines for medical treatment and its safety in the elderly". Geriatr Gerontol Int 2016;16:983-1001.

25. Filippatos TD, Makri A, Elisaf MS, et al. Hyponatremia in the elderly: challenges and solutions. Clin Interv Aging 2017;12:1957-65.

26. Juurlink DN, Mamdani MM, Lee DS, et al. Rates of hyperkalemia after publication of the Randomized Aldactone Evaluation Study. N Engl J Med 2004;351:543-51.

27. Lim LS, Fink HA, Blackwell T, et al. Loop diuretic use and rates of hip bone loss and risk of falls and fractures in older women. J Am Geriatr Soc 2009;57:855-62.

28. de Groot MH, van Campen JP, Moek MA, et al. The effects of fall-risk-increasing drugs on postural control: a literature review. Drugs Aging 2013;30:901-20.
29. Howard RL, Avery AJ, Slavenburg S, et al. Which drugs cause preventable admissions to hospital? A systematic review. Br J Clin Pharmacol 2007;63:136-47.

30. Renom-Guiteras A, Meyer G, Thürmann PA. The EU(7)PIM list: a list of potentially inappropriate medications for older people consented by experts from seven European countries. Eur J Clin Pharmacol 2015;71:861-75.

31. Olfson M, King M, Schoenbaum M. Benzodiazepine use in the United States. JAMA Psychiatry 2015;72:136-42.

32. Machado-Duque ME, Castaño-Montoya JP, MedinaMorales DA, et al. Association between the use of benzodiazepines and opioids with the risk of falls and hip fractures in older adults. Int Psychogeriatr 2018;30:941-6.

33. Lim YJ, Kim HY, Choi J, et al. Potentially Inappropriate Medications by Beers Criteria in Older Outpatients: Prevalence and Risk Factors. Korean J Fam Med 2016;37:329-33.

34. Al-Azayzih A, Alamoori R, Altawalbeh SM. Potentially inappropriate medications prescribing according to Beers criteria among elderly outpatients in Jordan: a cross sectional study. Pharm Pract (Granada) 2019;17:1439.

35. Prithviraj GK, Koroukian S, Margevicius S, et al. Patient Characteristics Associated with Polypharmacy and Inappropriate Prescribing of Medications among Older Adults with Cancer. J Geriatr Oncol 2012;3:228-37.

36. Fabbri E, Zoli M, Gonzalez-Freire M, et al. Aging and Multimorbidity: New Tasks, Priorities, and Frontiers for Integrated Gerontological and Clinical Research. J Am Med Dir Assoc 2015;16:640-7.

37. Achterhof AB, Rozsnyai Z, Reeve E, et al. Potentially inappropriate medication and attitudes of older adults towards deprescribing. PLoS One 2020;15:e0240463.

38. O'Mahony D, O'Sullivan D, Byrne S, et al. STOPP/ START criteria for potentially inappropriate prescribing in older people: version 2. Age Ageing 2015;44:213-8.

39. Reeve E, Gnjidic D, Long J, et al. A systematic review of the emerging definition of 'deprescribing' with network analysis: implications for future research and clinical practice. Br J Clin Pharmacol 2015;80:1254-68.

40. Garfinkel D, Ilhan B, Bahat G. Routine deprescribing of chronic medications to combat polypharmacy. Ther Adv Drug Saf 2015;6:212-33.

41. Bala SS, Chen TF, Nishtala PS. Reducing Potentially Inappropriate Medications in Older Adults: A Way Forward. Can J Aging 2019;38:419-33.

42. Weir DL, Lee TC, McDonald EG, et al. Both New and Chronic Potentially Inappropriate Medications Continued at Hospital Discharge Are Associated With Increased Risk 
of Adverse Events. J Am Geriatr Soc 2020;68:1184-92.

43. Wang P, Wang Q, Li F, et al. Relationship Between Potentially Inappropriate Medications And The Risk Of Hospital Readmission And Death In Hospitalized Older Patients. Clin Interv Aging 2019;14:1871-8.

44. Mekonnen AB, Redley B, de Courten B, et al. Potentially inappropriate prescribing and its associations with healthrelated and system-related outcomes in hospitalised older adults: A systematic review and meta-analysis. $\mathrm{Br} \mathrm{J}$ Clin Pharmacol 2021. [Epub ahead of print]. doi: 10.1111/ bcp. 14870 .

45. Cacchione PZ. Innovative care models across settings: Providing nursing care to older adults. Geriatr Nurs 2020;41:16-20.

(English Language Editor: J. Reynolds)
Cite this article as: Tian F, Liao S, Chen Z, Xu T. The prevalence and risk factors of potentially inappropriate medication use in older Chinese inpatients with multimorbidity and polypharmacy: a cross-sectional study. Ann Transl Med 2021;9(18):1483. doi: 10.21037/atm-21-4238 